\title{
Estudios de casos theory driven: inmigración y éxito escolar
}

\section{Resumen}

Este trabajo aborda la conexión entre el nivel empírico y el papel de la teoría en la investigación cualitativa. Se presentan dos investigaciones sobre los procesos de persistencia académica en estudiantes de familias de origen migratorio; informes nacionales e internacionales, y resultados de investigaciones previas que indican que estos jóvenes tienen el doble de posibilidades de abandonar el sistema educativo. Los estudios de casos desarrollados con orientación theory driven sirven de base para mostrar el uso de instrumentos cuantitativos y cualitativos integrados en un diseño mixto secuencial y ejemplificar algunos análisis de la información cualitativa.

\section{Palabras clave}

Estudio de caso; análisis cualitativo; inmigración; análisis de redes; éxito escolar; resiliencia doi: 10.11144/Javeriana.m11-22.ectd

María Paz Sandín-Esteban Universidad de Barcelona, España mpsandin@ub.edu https://orcid.org/0000-0003-1836-6126

Para citar este artículo / To cite this article

Sandín-Esteban, M. P. (2018). Estudios de casos theory driven: inmigración y éxito escolar. magis, Revista Internacional de Investigación en Educación, 11 (22), 57-74. doi: 10.11144/Javeriana.m11-22.ectd

\begin{abstract}
This paper addresses the connection between the empirical endeavor and the role of the theory in the qualitative research. Two researches on the academic persistence processes in students of migrant-background families are provided. National and international reports are provided as well as the results from previous researches showing that these students are twice likely to drop out the school system. The study cases conducted on a theory-driven basis are drawn upon to show the use of quantitative and qualitative instruments blended into a sequential mixed design and to illustrate some qualitative information analyses.
\end{abstract}

\section{Keywords}

Study case; qualitative analysis; immigration; network analysis; school success; resiliency 


\section{Introducción}

La investigación cualitativa es una poderosa forma de dar luz a los fenómenos estudiados, explorar y comprender la realidad. Pero su valor también reside en contribuir a la construcción teórica; se llega a afirmar que, en este enfoque, la teoría es el producto más importante (Morse, 2003).

Este reconocimiento se produjo hace ya casi tres décadas, cuando en el marco histórico de transición y emergencia de criterios de rigor científico propios de la investigación cualitativa, se defendió el valor de los estudios cualitativos para contribuir a la mejora de la teoría y aparecieron propuestas para su evaluación que incluían referencias explícitas a indicadores y procedimientos de construcción teórica. Por ejemplo, Nancy Burns (1989) consideró que el resultado del análisis de los datos cualitativos es un esquema teórico que otorga significado al fenómeno en estudio y propuso cinco estándares de calidad para la investigación cualitativa: viveza descriptiva, congruencia metodológica, precisión analítica, conectividad teórica y relevancia heurística. En la misma línea, Janice M. Morse (1997) defendió que la calidad de una investigación cualitativa no debe limitarse a los aspectos de rigor metodológico, sino que deben valorarse también los resultados teóricos de la investigación per se y planteó una serie de cuestiones relacionadas, por ejemplo, con la estructuración del contenido empírico desde la teoría, el encaje entre las categorías de análisis y los datos, la plausibilidad de las conexiones macro-micro, y la relación entre la teoría y su aplicación práctica. Su propuesta incluye seis criterios para examinar la teoría derivada de la investigación cualitativa: claridad, estructura, coherencia, alcance, generalización y utilidad pragmática.

En las últimas décadas, la investigación cualitativa ha alcanzado una madurez considerable. Quizá podamos nombrar como obra de referencia el Handbook of Qualitative Research, editado por primera vez en 1994 por Norman K. Denzin e Yvonna S. Lincoln y que en 2017 se ha publicado en su quinta edición con una amplia revisión y actualización de contenidos. A pesar de la aceptación y desarrollo de la investigación cualitativa en diversas disciplinas de las Ciencias Sociales; de los tipos de conocimiento que proporcionan diversos enfoques, entre otros, etnográfico, narrativo, fenomenológico, teoría fundamentada y estudio de caso (Creswell, 2007); e incluso disponiendo de un amplio abanico de procedimientos analíticos para la elaboración conceptual (Coffey \& Atkinson, 1996), el aporte a la generación de conocimiento quizá sea una de sus facetas con menor recorrido. Se llega a decir que la investigación cualitativa es larga en datos y corta en teorías (Morse, 2003).

Para ejemplificar la discusión sobre el uso de la teoría en investigación cualitativa y el rol que ocupa, especialmente cuando se analizan los datos, en este artículo se presentarán y discutirán algunos procedimientos analíticos utilizados en el marco de dos investigaciones sobre el éxito escolar en alumnado de origen migratorio. En primer lugar, de manera sucinta, se expondrán algunas controversias y diversas miradas sobre el papel de la teoría en la investigación cualitativa para, después, presentar las preguntas de investigación que tratan de responder los estudios desarrollados, el contexto de aplicación y su diseño general. Más concretamente, se caracterizarán como estudios de casos theory driven.

\section{Investigación cualitativa y generación de teoría}

La contribución teórica en los estudios cualitativos no ha estado exenta de discusión en la literatura, vinculada especialmente a un aspecto clave 
de las investigaciones: el análisis de los datos; hay distintas visiones con relación al nivel de interpretación que cabría esperar, que Anselm Strauss y Juliet Corbin (1990) sintetizan en tres posturas generales caracterizadas por diferentes grados de elaboración y abstracción teórica (figura 1). Precisamente, de forma reciente, una edición especial de la revista magis ahondó en el desarrollo y las posibilidades del análisis de datos en la investigación cualitativa con trabajos acerca de la noción misma de comprensión y la atribución de sentido por parte del investigador (Medina, 2014) y sobre la relación dinámica entre los marcos de comprensión propios del investigador y aquellos más cercanos a los participantes de una realidad educativa (Guzmán-Valenzuela, 2014).

Figura 1

Niveles de interpretación en la investigación cualitativa

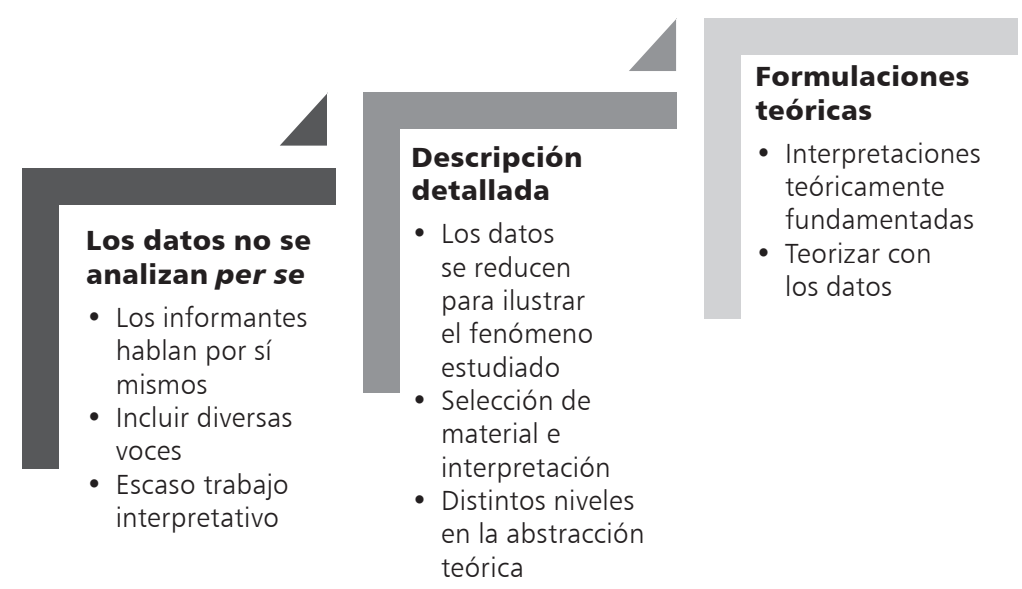

Fuente: elaboración propia, basado en Anselm Strauss y Juliet Corbin (1990)

Para Kathleen M. Eisenhardt y Melissa E. Graebner (2007, p. 25), la construcción de teoría a partir de estudios de caso es "uno de los mejores puentes (si no el mejor) entre la rica evidencia cualitativa y la corriente principal deductiva de investigación". Los dos enfoques de estudio de caso ya bien establecidos en la literatura son el deductivo, representado en el trabajo de Robert K. Yin (1994), que sigue una lógica de replicación/confirmación de la teoría a través de los casos; y el inductivo, desarrollado inicialmente por Barney G. Glaser y Anselm L. Strauss (1967) en su formulación de la Teoría Fundamentada con énfasis en el descubrimiento y desarrollo teórico a partir de los datos empíricos. Con estos dos marcos de referencia principales para el desarrollo de estudios de caso, los estudios de caso de orientación cualitativa se enfrentan a tensiones entre una epistemología idiográfica y las expectativas de que sus resultados puedan validar o ampliar un cuerpo sustantivo de conocimiento. Las metodologías integradoras que aúnan los enfoques analíticos deductivo e inductivo en la investigación cualitativa (Meyer \& Ward, 2014), reconociendo la posibilidad tanto del contraste teórico como de la emergencia teórica, alivian estas tensiones. Por ejemplo, Harm-Jan Steenhuis y Erik J. de Bruijn (2006) combinan en un método de "estudio progresivo de casos", las fortalezas de ambos enfoques, con mayor alineación con el inductivo; en su propuesta, 
la literatura no se analiza al inicio para generar un marco conceptual con fines de prueba, ni tampoco se descarta totalmente utilizar una teoría substantiva, sino que sensibiliza al investigador sobre áreas de interés y lo hace consciente del estado del arte; se utilizan los principios de la teoría fundamentada para desarrollar nueva teoría y el muestreo de bola de nieve para la selección de casos; también se manejan técnicas de validación al contrastar determinada información en los casos posteriores, es decir, con lógica de replicación.

Añadido a los intentos tímidos de construcción teórica, los investigadores se enfrentan a dificultades para comunicar los resultados de sus estudios en los medios de difusión científica. Caroline Bradbury-Jones, Julie Taylor y Oliver Herber (2014) señalan que en un contexto cada vez más exigente para la publicación de aportaciones científicas, dar cuenta del proceso de construcción conceptual en la investigación cualitativa y de la compleja relación entre teoría y método no es una tarea sencilla. Sobre el argumento de que la ausencia de teoría en una investigación cualitativa debilita su calidad, estos autores han analizado en qué medida la teoría aparece en los artículos publicados en cinco revistas internacionales relevantes del ámbito de la salud, medicina y ciencias sociales y han planteado una tipología de cinco niveles de visibilidad teórica que abarca el uso de la teoría en un rango desde el nivel 1 (la teoría aparentemente no aparece) hasta el nivel 5 (donde la teoría se aplica y es totalmente visible a lo largo de todo el proceso de investigación). De los 55 artículos analizados, solo 9 hacían uso de la teoría según el último nivel; los autores señalan que en estos estudios los investigadores podrían haber utilizado la teoría, pero no haberlo comunicado claramente en el artículo. La tipología de niveles de visibilidad teórica (tabla 1) ofrece un marco para valorar la fuerza teórica de un estudio siendo de utilidad a los propios investigadores, por ejemplo, en la preparación de la publicación de sus manuscritos, y también para las personas que deben evaluar estos trabajos.

Tabla 1

Tipología de niveles de visibilidad teórica

\begin{tabular}{l|l}
\hline Nivel de visibilidad teórica & Descriptor \\
\hline Nivel 1: Aparentemente ausente & La teoría no se menciona. \\
\hline Nivel 2: Implícita & $\begin{array}{l}\text { La teoría se menciona o discute brevemente (en la introducción } \\
\text { normalmente o como marco general) y es posible que se mencione a } \\
\text { teóricos en el campo, sin referencia explícita a su influencia en el estudio. }\end{array}$ \\
\hline Nivel 3: Parcialmente aplicada & $\begin{array}{l}\text { Los investigadores explícitamente sitúan su estudio en una teoría particular, } \\
\text { pero abandonan los esfuerzos por relacionar, aplicar o interpretar los } \\
\text { resultados en ese contexto. } \\
\text { La teoría se usa solo parcialmente a lo largo del proceso de investigación en } \\
\text { relación con los objetivos, preguntas de entrevista o el análisis de datos. }\end{array}$ \\
\hline Nivel 4: Aplicada retrospectivamente & $\begin{array}{l}\text { La teoría se considera al final del estudio para dar sentido a los resultados } \\
\text { de investigación. } \\
\text { La teoría se introduce de manera retrospectiva al final del estudio. }\end{array}$ \\
\hline Nivel 5: Consistentemente aplicada & $\begin{array}{l}\text { La teoría se aplica consistentemente a lo largo de todo el proceso } \\
\text { de investigación. } \\
\text { La teoría guía y dirige las diversas fases del proceso de investigación y } \\
\text { puede ser reconocida a lo largo de un artículo publicado. }\end{array}$ \\
\hline \hline
\end{tabular}

Fuente: Caroline Bradbury-Jones, Julie Taylor y Oliver Herber (2014, p. 137) 
Situados hasta aquí las cuestiones y debates esenciales en torno a la investigación cualitativa y la generación de teoría, presentamos en los siguientes apartados dos investigaciones de estudio de casos sobre el éxito escolar del alumnado de procedencia migratoria, para definir su "contexto teórico" (estudios theory driven) en estrecha relación con las cuestiones de investigación, y focalizando en algunos procesos de análisis de la información cualitativa desarrollados con la finalidad de ilustrar las decisiones tomadas y el tipo de resultados obtenidos. Siguiendo la aportación de Bradbury-Jones, Taylor y Herber (2014) destacaremos el papel que ha jugado la teoría en su diseño y desarrollo y mostraremos algunos ejemplos de enfoque deductivo e inductivo de análisis de la información cualitativa obtenida.

\section{Área temática, contexto y diseño}

El período de la Educación Secundaria Obligatoria' constituye una etapa de alto riesgo o vulnerabilidad académica para la población inmigrante. Así lo recogen diversos informes nacionales e internacionales que ponen de relieve que en estos colectivos los estudiantes tienen más probabilidades de abandonar sus estudios (Boada, Herrera, Mas, Miñarro, Olivella, Riudor, Calero, Choi \& Escardíbul, 2011; OECD, 2011; Serra \& Palaudàrias, 2010) en su tránsito a la educación postobligatoria².

Teniendo presente la complejidad de esta realidad, las preguntas de investigación que enmarcaron nuestros trabajos fueron cualitativas en el sentido que Carmen de la Cuesta-Benjumea (2008) confiere a las "preguntas cualitativas" cuando se quiere comprender un área de la que se sabe poco o no convence lo que se sabe, cuando se trata de comprender situaciones complejas en las que se contemplan variables culturales o cuando se desea conocer cómo las personas de un grupo experimentan un proceso, y comprender en profundidad y con detalle un fenómeno. De forma general, nos preguntábamos ipor qué es tan elevado el nivel de abandono escolar en la etapa postobligatoria?, ¿cuáles son los procesos educativos y sociales que favorecen o limitan la persistencia académica en estos jóvenes? En nuestras investigaciones, analizamos concretamente sus trayectorias académicas en el tránsito de la enseñanza secundaria obligatoria (ESO) a la enseñanza secundaria postobligatoria (PO); estudios previos han constatado que el porcentaje de abandono entre estas etapas es muy elevado y es el doble entre los jóvenes de procedencia migratoria. En este marco se han realizado dos investigaciones de carácter longitudinal (figura 2) que se iniciaban con una primera caracterización de los estudiantes en el último curso de la enseñanza obligatoria (ESO, fase 1) continuando con el seguimiento de su incorporación a la enseñanza postobligatoria y permanencia en esta etapa en el siguiente curso académico ( $\mathrm{PO}$, fase 2 ).

1 La Educación Secundaria Obligatoria (ESO, de 4 años de duración) hace referencia a la educación secundaria en España (desde 1996) y tiene por objeto la preparación de estudiantes entre 12 y 16 años de edad para realizar estudios posteriores y/o su integración en el mundo laboral.

2 El estudiante debe obtener el certificado de ESO antes de continuar con la elección de una opción formativa en la etapa postobligatoria que tiene 2 años de duración. 
Figura 2

Diseño longitudinal general de las dos investigaciones con estudio de casos

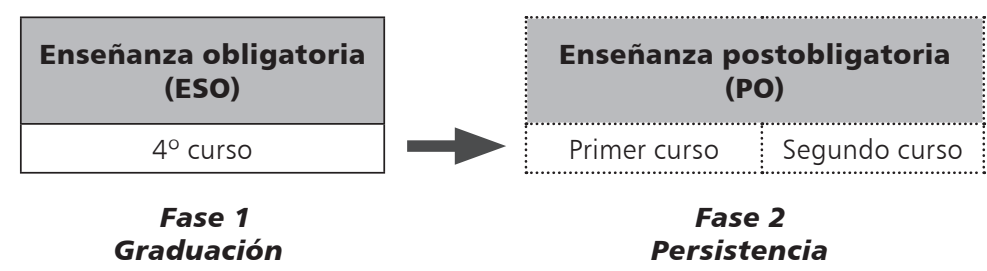

Fuente: elaboración propia

Ambas investigaciones se realizaron con la colaboración de varios Institutos de Enseñanza Secundaria formando parte todos los estudiantes de familias de procedencia extranjera matriculados en el último curso de la ESO, independientemente de su origen cultural (primer estudio $n=94$, segundo estudio $n=87$, estudiantes entre 15 y 18 años). El método respondió a la modalidad de "estudio de casos de tipo instrumental" en la que los casos particulares se analizan para obtener mayor comprensión sobre una temática o refinar una teoría (Stake, 1995). En ambos estudios, en la primera fase, se implementan diversos instrumentos de orientación cuantitativa (QUAN) y se introducen secuencialmente según los objetivos específicos de cada estudio las estrategias de tipo cualitativo en la segunda fase ( $\rightarrow$ qual).

\section{Estudios de casos theory driven - investigación con contexto teórico}

Sobre el lugar que ocupa la teoría en la investigación social se reconocen en la literatura dos aproximaciones dominantes: teoría primero o teoría después, verificación de teoría o generación de teoría, enfoques theory-driven y grounded theory (Punch, 2005). Un enfoque theory driven utiliza la teoría a priori para diseñar la investigación e implica una lógica deductiva; la investigación theory driven presenta un claro contexto teórico que orienta las cuestiones y los datos a obtener, la teoría no es un fin en sí mismo sino que orienta la investigación empírica. Por el contrario, en los enfoques de teoría fundamentada, la teoría no dirige el diseño o desarrollo de la investigación (al menos en las fases iniciales), sino que adoptan un enfoque prioritariamente inductivo; utilizan los datos para explorar posibilidades teóricas, se orientan al desarrollo teórico.

En nuestro caso, el análisis bibliográfico inicial sobre persistencia académica e inmigración, descubrió dos cuestiones clave que nos abocaron a la indagación de marcos teóricos explicativos de forma que algunas de las teorías estudiadas contribuyeron finalmente al planteamiento de nuestras propias investigaciones: por un lado, la paradoja entre las altas expectativas académicas de los estudiantes y sus familias, y la baja consecución de las mismas; es decir, la existencia de una voluntad individual y familiar de progreso educativo, que no acababa de alcanzarse; por otro, la constatación de que algunos de estos jóvenes - a pesar de las circunstancias de desventaja académica o social - presentaban trayectorias continuistas, lo cual contrasta con la experiencia generalizada de abandono de sus iguales. Consideramos entonces que era necesario indagar en esta diferenciación educativa y explorar los resortes que la promueven. Este enfoque de la situación superaba los discursos y estudios centrados en las "limitaciones o déficits" de los estudiantes y adoptaba una visión "positiva" del fenómeno, para destacar sus potencialidades y visibilizar la existencia de trayectorias de éxito. 
Los estudios de casos que presentamos brevemente a continuación se orientaron desde una perspectiva theory-driven para explorar y comprender los procesos de escolarización y éxito escolar de los estudiantes en el sistema educativo con una postura integradora considerando que nuestros trabajos comienzan con teoría, pero también pueden aportar a la generación teórica (Meyer \& Ward, 2014).

Primer estudio: Resiliencia y persistencia académica

En los últimos años, los trabajos conceptuales y empíricos sobre la resiliencia han tomado relevancia a la hora de analizar por qué algunos estudiantes tienen éxito en el sistema educativo, a diferencia de otros iguales del mismo contexto o comunidad social (Bartolomé-Pina, 2017). Las teorías y modelos sobre la resiliencia constituyeron el contexto teórico de esta primera investigación. Se sostuvo la hipótesis de que los estudiantes que superaban la transición académica de riesgo ESO-PO presentaban características específicas de resiliencia, al conjugar capacidades individuales y apoyos del entorno. La resiliencia es un término que proviene de la física y se utiliza para describir aquellos materiales que, a pesar de estar sometidos a situaciones de desgaste $u$ otras agresiones, consiguen recuperar su estado original. En la literatura científica, este concepto se ha tratado de diferentes maneras (Fergus \& Zimmerman, 2005; Kaplan, 1999; Luthar, 2003; Masten, 2001) con cierta unanimidad en considerarla una "competencia efectiva de afrontamiento" como respuesta a una situación de riesgo o adversidad.

Figura 3

Contextos teóricos de los estudios desarrollados y cuestiones de investigación

\section{Preguntas generales de investigación}

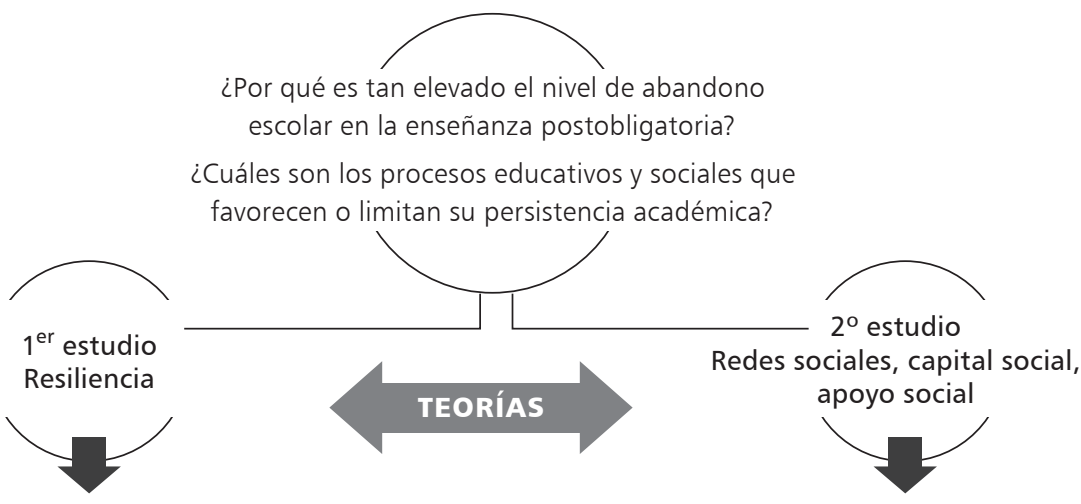

\section{Cuestiones de investigación}

- ¿Qué contextos de vulnerabilidad enfrenta el alumnado?

- ¿Cuáles son los factores protectores que pueden incidir en trayectorias de éxito?

¿Difieren según grupos culturales?

- ¿En qué medida pueden incidir los programas y acciones educativas?

- ¿Cómo es la relación entre la percepción de necesidad y el apoyo recibido? ¿Está mediada culturalmente?

\section{Cuestiones de investigación}

- ¿Cuáles son la estructura, la composición y la funcionalidad de las redes personales de los estudiantes?

- ¿Cuál es su concepción de apoyo social?

- ¿Qué carencias de apoyo señalan en su entorno escolar, personal, familiar y social?

- ¿Qué vínculos prosociales favorecen las trayectorias continuistas?

- ¿Cuáles son sus expectativas académicas y profesionales? 
El objetivo principal consistió en analizar la relación entre la resiliencia y el éxito escolar del alumnado, entendido como persistencia y no abandono del sistema educativo en la transición ESO-PO y mantenimiento durante el primer año de la postobligatoria. En la fase 1 (figura 2) se aplicaron tres instrumentos: un cuestionario general sobre información personal, familiar, datos sociodemográficos, amistades, recursos de apoyo en los centros educativos, expectativas y autopercepción académica; se adaptó y utilizó la escala de resiliencia SV-RES (Saavedra-Guajardo \& Villalta-Paucar, 2008) que permite obtener una puntuación global de resiliencia, además de diferentes puntuaciones según 12 factores (tabla 2); y una escala de centro, que permitió recoger información sobre su percepción del centro escolar, el profesorado, la relación familia-escuela, y la integración social entre compañeros. El análisis de esta información en la primera fase descubrió la relación entre la resiliencia y diversas variables de interés en la investigación (procedencia, percepción de logro, referentes de éxito). El seguimiento individual de los estudiantes durante su transición al siguiente curso académico, fase 2, constatando su permanencia o abandono de los estudios, nos permitió valorar si existía una relación entre resiliencia y persistencia académica: se obtuvo que la resiliencia estaba relacionada con las trayectorias continuistas de los estudiantes, aquellos que abandonaron los estudios al finalizar la etapa obligatoria o en los inicios de la postobligatoria mostraron niveles más bajos y significativos de resiliencia según la escala adaptada SV-RES (Sandín-Esteban \& Sánchez-Martí, 2013).

Tabla 2

Factores de resiliencia de la escala SV-RES

\begin{tabular}{l|l|l|l|l}
\hline $\begin{array}{l}\text { Competencias } \\
\text { interaccionales } \\
\text { de Edith Grot- } \\
\text { berg (1995) }\end{array}$ & \multicolumn{2}{|l}{ Niveles de estructuración de la conciencia (Saavedra \& Villalta, 2008) } \\
\cline { 2 - 4 } & $\begin{array}{l}\text { Condiciones } \\
\text { de base }\end{array}$ & $\begin{array}{l}\text { Visión de } \\
\text { uno mismo }\end{array}$ & $\begin{array}{l}\text { Visión del } \\
\text { problema }\end{array}$ & $\begin{array}{l}\text { Respuesta } \\
\text { resiliente }\end{array}$ \\
\hline Yo soy/Yo estoy & F1: Identidad & F2: Autonomía & F3: Satisfacción & F4: Pragmatismo \\
\hline Yo tengo & F5: Vínculos & F6: Redes & F7: Modelos & F8: Metas \\
\hline Yo puedo & F6: Afectividad & F10: Autoeficacia & F11: Aprendizaje & F12: Generatividad \\
\hline \hline
\end{tabular}

Fuente: basado en Eugenio Saavedra-Guajardo y Marco Villalta-Paucar (2008)

En la etapa postobligatoria, tras la transición exitosa, se realizó una selección de casos resilientes que se abordaron con técnicas cualitativas. Con una orientación theory driven a lo largo de toda la investigación, el diseño de las entrevistas: a) contempló dimensiones conceptuales a partir de los modelos y teorías sobre resiliencia, también sobre los procesos migratorios, de integración social y educativa; b) incorporó la exploración de algunas áreas no previstas inicialmente atendiendo a los resultados de otros estudios, y del análisis inicial en la primera fase y c) se introdujeron preguntas catalizadoras (Resilience Research Center, s.f.) de utilidad para hacer emerger narrativas sobre resiliencia y explorar el significado particular que algunos conceptos como "éxito" o "riesgo" adquieren desde un punto de vista personal o familiar, en distintas culturas y en diferentes contextos. Para el análisis de las entrevistas se adoptaron dos enfoques prioritarios, como se muestra en la figura 4 (intracaso deductivo y transversal temático), pero también exploramos otros enfoques y procedimientos analíticos que expondremos más adelante (comparaciones constantes Teoría fundamentada-y metáforas). 


\section{Cuestionario Redes 1 Enseñanza obligatoria}

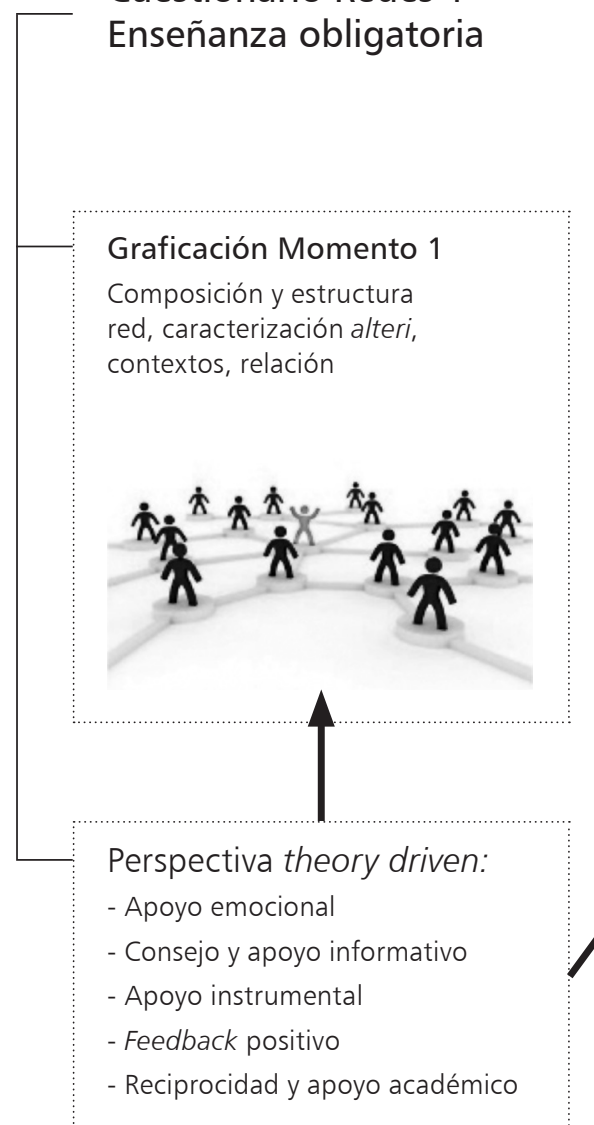

\section{Entrevista Egonarrativa.} Postobligatoria

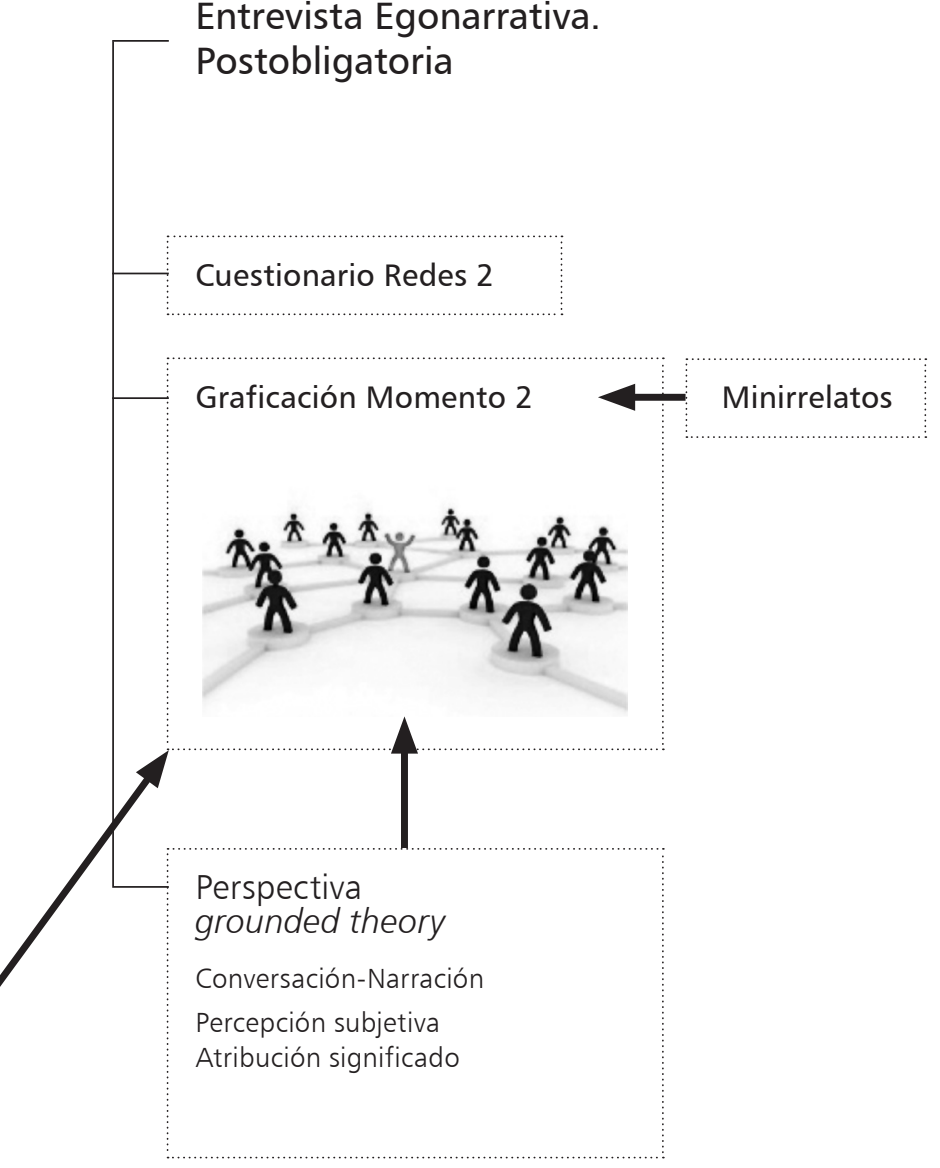

Fuente: elaboración propia

La primera estrategia consistió en un análisis intracaso de manera que en cada entrevista se identificaron los fragmentos que conceptualmente respondían a las dimensiones y factores de resiliencia propuestos por Eugenio Saavedra-Guajardo y Marco Villalta-Paucar (2008). Cada caso constituía una historia de éxito, lo que permitía el contraste con la teoría y mostraba una combinación particular y única de factores. En la siguiente tabla, a modo de ejemplo, se muestra un extracto reducido de la matriz utilizada en el análisis de dos casos para la dimensión "respuesta resiliente", que incluye los factores de resiliencia pragmatismo (F4), metas (F8) y generatividad (F12); en la matriz se realiza una estimación del nivel de significación de los factores y se indica su presencia (+) o ausencia (-) en el caso; se presentan algunas evidencias narrativas y se realiza una valoración global del caso en la dimensión analizada. 
Tabla 3

Ejemplo de análisis de entrevista (extracto de un caso). Estrategia 1

Dimensión Respuesta resiliente SV-RES

Consiste en los siguientes factores: Pragmatismo (F4), pragmatismo en la interpretación de las acciones realizadas;

Metas (F8), capacidad de actuar para enfrentarse a una situación problemática; y Generatividad (F12), capacidad de pedir ayuda a los demás para resolver situaciones difíciles.

\begin{tabular}{|c|c|c|c|c|c|}
\hline \multirow[t]{2}{*}{ Entrevista } & \multirow{2}{*}{$\begin{array}{l}\text { Extractos analíticos } \\
\text { de cada caso }\end{array}$} & \multicolumn{3}{|c|}{$\begin{array}{l}\text { Factores de } \\
\text { ponderación }\end{array}$} & \multirow[t]{2}{*}{ Valoración global } \\
\hline & & F4 & F8 & F12 & \\
\hline $1-1$ & $\begin{array}{l}\text { Establecimiento de metas; se observan } \\
\text { algunas concretas, como estudiar Derecho. } \\
\text { Pero también se observan dudas ("si entro } \\
\text { en la Facultad de Derecho...") y falta de } \\
\text { concreción ("para llegar donde quiero } \\
\text { llegar" [... "y todo eso"). } \\
\text { La elección de los estudios se realiza } \\
\text { teniendo en cuenta la valoración de sus } \\
\text { capacidades y oportunidades ("si veo } \\
\text { que no puedo, haré otra cosa, como } \\
\text { trabajo social"). } \\
\text { La evaluación pragmática de su situación } \\
\text { actual ("ahora mismo no puedo hacer } \\
\text { mucho" o "solo con la ESO no se puede } \\
\text { hacer mucho"). }\end{array}$ & + & - & - & $\begin{array}{l}\text { Si bien los factores "generatividad" y } \\
\text { "metas" están presentes en el relato, no } \\
\text { parecen ser decisivos en el proceso de } \\
\text { resiliencia: no se expresan con la suficiente } \\
\text { convicción, debido a las dudas sobre } \\
\text { la posibilidad de conseguir esas metas } \\
\text { y la falta de concreción a largo plazo. } \\
\text { Prevalece una valoración pragmática de su } \\
\text { presente y proyección futura. }\end{array}$ \\
\hline $1-3$ & $\begin{array}{l}\text { Se declara un plan de acción sobre la } \\
\text { manera de conseguir sus metas, así como } \\
\text { ("lo primero es llegar, conocer gente, } \\
\text { integrarse, pedir ayuda a los demás y a los } \\
\text { profesores, y así es como se puede salir } \\
\text { adelante", "me tomé el cuarto año de ESO } \\
\text { como un juego, para disfrutar del año } \\
\text { aprendiendo"). } \\
\text { Hay metas claras ("quiero estudiar } \\
\text { psicología", "quiero abrir mi propia consulta } \\
\text { y estar con mi familia", "ayudar a los } \\
\text { demás", "puedo hacer algo, por ejemplo, } \\
\text { con personas que tienen miedo de hacer } \\
\text { algo, les puedo ayudar a que no tengan } \\
\text { miedo"). }\end{array}$ & - & + & + & $\begin{array}{l}\text { No hay ningún indicador evidente } \\
\text { del pragmatismo predominante en } \\
\text { la interpretación de sus acciones. Los } \\
\text { factores "Metas" y "Generatividad" se } \\
\text { expresan en relación con algunos aspectos } \\
\text { de desarrollo personal y profesional. Sí } \\
\text { es evidente una clara formulación de las } \\
\text { metas y de los objetivos, expresados de } \\
\text { manera proactiva. }\end{array}$ \\
\hline
\end{tabular}

Fuente: elaboración propia

En este enfoque analítico, sin abandonar una postura exploratoria o de descubrimiento, predomina el análisis de los casos en su encaje con las dimensiones y la teoría, se analizan desde una lógica deductiva que contribuye a la contrastación y validación teórica (Yin, 1994).

El análisis intracaso constató, entre otras cuestiones, que la presencia o ausencia de un factor tomada de forma aislada no explica por sí misma el perfil resiliente; hay distintas historias de resiliencia en las que los factores se combinan de forma sinérgica y única; la presencia de un mayor o menor número de factores significativos en el caso, aunque importante, no era decisiva. La segunda estrategia utilizada en el análisis de las entrevistas, complementaria a la anterior, consistió en un análisis temático guiado por las dimensiones de la entrevista en el que se realizó una síntesis transversal (Yin, 1994) que permitió identificar similitudes y diferencias en las narrativas de éxito y garantizar una comprensión holística de las experiencias de éxito y vulnerabilidad de los jóvenes. Esta estrategia de análisis de contenido a través de categorías es ampliamente utilizada en los estudios cualitativos. 
El valor y riqueza de los datos obtenidos en las entrevistas en profundidad nos llevó a explorar una tercera aproximación de análisis no tan extendida, de tipo inductivo, para aplicar los procedimientos de la Teoría Fundamentada. Así descubrimos una variable que no había sido contemplada intencionalmente en nuestro estudio: "el tiempo". Ante la existencia de factores de riesgo en el ámbito personal, familiar o escolar de los jóvenes aparecían factores protectores que mitigaban su impacto; hasta aquí nada nuevo que no se haya discutido en los modelos de resiliencia sobre la combinación de factores protectores-factores de riesgo. Lo relevante, lo que emergió de los datos (Trinidad-Requena, Carrero-Planes \& Soriano-Miras, 2006) fue un patrón de tiempo entre la aparición de un tipo y otro de factores: no era en "cualquier tiempo" que el factor protector acontecía, sino que aparecía "pronto", en diversas ocasiones y circunstancias: familiares en el país de origen que logran conseguir el dinero necesario para un viaje de reagrupación familiar ("mi madre llegó enseguida"), personas en el país de llegada que ofrecen un contrato laboral ("no había pasado ni una semana y mi madre ya consiguió un trabajo"), aprendizaje exitoso de una segunda lengua en el ámbito escolar ("en un mes yo ya sabía hablar catalán"); en términos de los procedimientos generales de análisis en teoría fundamentada, emergía una posible "categoría central" que nos permitía dar nueva luz al fenómeno de la resiliencia en estos jóvenes: "el tiempo cuenta" constituía una elaboración conceptual iluminativa (Morse, 1997).

La categoría central suele responder a la pregunta: ¿cuál es el suceso principal?, puesto que en esa categoría se condensa la esencia del modo de comportamiento de las personas estudiadas. La categoría central es la que tiene un mayor poder explicativo y predomina sobre las otras categorías (Andréu-Abela, García-Nieto \& Pérez-Corbacho, 2007, p. 71).

Otra de las formas de teorización en la investigación cualitativa son las metáforas (Gibbs, 2012). Las metáforas responden a estructuras de conocimiento humano, permiten mapear o proyectar una experiencia en términos de otra experiencia; comunican lo abstracto por medio de términos concretos y ayudan a reducir la complejidad y la ambigüedad (Lakoff \& Johnson, 2004). Permiten adquirir una comprensión de temas complejos o situaciones nuevas a través de conceptos conocidos. Susan Llewellyn (2003) contempla las metáforas como una de las cinco formas disponibles de teorización en la investigación cualitativa; señala que son una herramienta poderosa para teorizar experiencias a partir de estudios cualitativos que, sin embargo, ha sido poco desarrollada. En nuestro trabajo sobre la resiliencia en estudiantes de procedencia migratoria en situación de vulnerabilidad, "el cactus como metáfora" (original de Milton H. Erickson) mostraba ser una forma válida de expresar y revelar el conocimiento sobre la experiencia de resurgir ante la adversidad; los cactus son plantas capaces de subsistir en cualquier condición: con agua escasa, apartadas de la luz, con poca tierra, resisten; el cactus ofrece una imagen desafiante, espinosa, como las situaciones que enfrentan estos jóvenes en sus procesos vitales, pues logran avanzar en su desarrollo personal, social y académico, y resurgen, como finalmente también florece el cactus, con gran belleza. Aunque el cactus es muy capaz y se adapta enormemente, su resistencia no es eterna, y sin algunos cuidados "a tiempo", no logrará sobrevivir, como aquellos estudiantes que abandonarán los estudios.

\section{Segundo estudio: Redes personales}

Recientemente, Alan J. Richardson (2017) sitúa la construcción teórica en los estudios cualitativos en la discusión sobre el conocimiento acumulativo, tradicionalmente reconocido a la investigación positivista. En investigación cualitativa, nos dice, el conocimiento no es aditivo en un sentido algebraico, sino discursivo, y refleja una mayor extensión y densidad de las relaciones intertextuales en un campo, una red discursiva más amplia; propone una taxonomía para diseñar y dar cuenta de las investigaciones a partir de dos ejes: la extensión de nuestro conocimiento (expandir los períodos de tiempo, el contexto, y/o la perspectiva usada para explorar un fenómeno), y la profundidad de nuestro conocimiento (nuevo conocimiento empírico, pluralismo metodológico — por ejemplo, el uso de métodos mixtos secuenciales para generar contribuciones progresivas-, elaboración teórica o generalización analítica).

La segunda investigación realizada sobre las trayectorias de permanencia en el sistema educativo supuso una extensión del conocimiento, en dos sentidos, por un lado, al ampliar el seguimiento de los estudiantes a tres cursos académicos, desde el último año de la educación obligatoria hasta finalizar las opciones postobligatorias (bachillerato o ciclo formativo), y por otro, al situar la comprensión del éxito educativo en niveles explicativos poco explorados hasta el momento como el contexto "meso" (las redes de relaciones de los estudiantes), el cual constituye un horizonte articulador entre los elementos habitualmente adscritos a las características psicoeducativas y circunstancias del alumnado ("nivel micro") y una orientación más contextual vinculada a factores de la estructura social ("nivel macro"). Desde esta perspectiva, poco explorada y novedosa en el estudio de las trayectorias académicas de los estudiantes de procedencia migratoria, 
se sostiene que el sistema relacional de los jóvenes es fundamental en sus procesos de integración, una oportunidad para la participación social y la promoción personal. La adopción de esta perspectiva responde también al eje de profundidad por cuanto el nuevo conocimiento empírico adquirido en el estudio previo mostró que la dimensión resiliente más relevante en los estudiantes de éxito era el "yo tengo" del modelo de Edith Grotberg (1995) referida a los soportes de vínculos, redes y modelos de referencia. Así, este segundo estudio daba continuidad al objetivo de comprender las trayectorias vitales de los estudiantes, ahora explorando en qué medida sus redes personales pueden constituir un factor promotor o protector de sus trayectorias académicas que incide en su persistencia y éxito escolar.

Por la novedad del enfoque y manteniendo una orientación theory driven para el diseño y desarrollo de la investigación, en este estudio también fue esencial disponer de un marco conceptual de referencia. Se llevó a cabo un estudio sistemático de la literatura, que cubrió un período inicial de 12 años, y una revisión e integración de las actuales conceptualizaciones sobre los constructos de "capital social" y "apoyo social". Ambos resaltan los espacios relacionales y el sistema de apoyo social como favorecedores de la integración y del éxito en los procesos migratorios (García-Ramírez, Martínez-García \& Albar-Marín, 2002; Stanton-Salazar, 1997). Samantha Meyer y Paul Ward (2014) señalan que a los conceptos teóricos hay que acceder empíricamente con los métodos apropiados. En este sentido, fue fundamental complementar la elaboración del contexto teórico de este estudio (figura 5) con la perspectiva del Social Network Analysis (ARS en español) (Scott \& Carrington, 2011), un enfoque amplio que ha desarrollado procesos metodológicos y de creación de instrumentación propios para la medición, análisis y descripción de las estructuras, patrones y formas de interacción que emergen de los vínculos entre actores sociales diversos (personas, organizaciones, etc.) (Molina, 2001).

Figura 5

Contexto teórico del estudio de redes personales

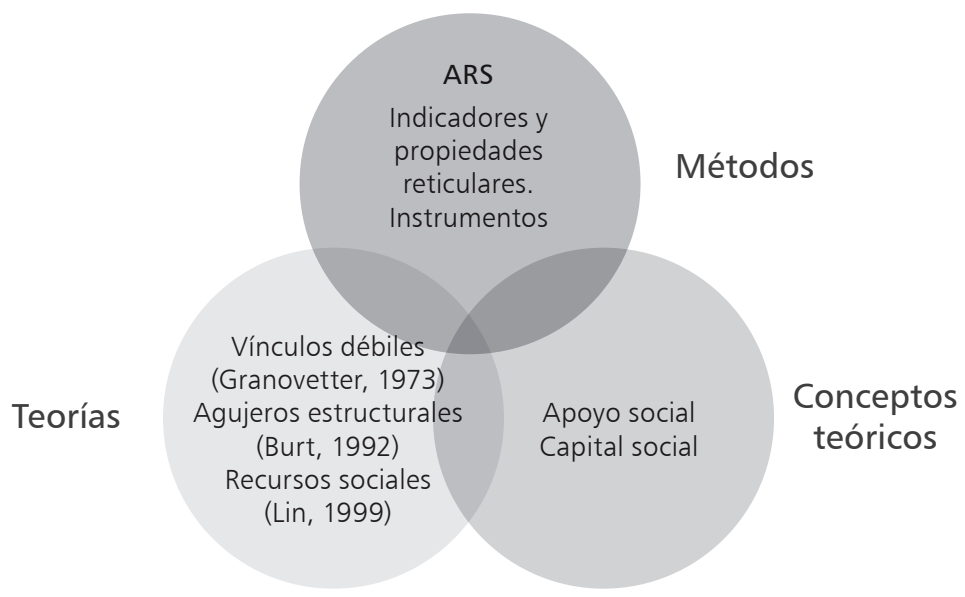

Fuente: elaboración propia

Desde este marco conceptual, el estudio se inicia con el establecimiento de las redes personales de 87 estudiantes en el último curso de la enseñanza obligatoria a través de dos cuestionarios de redes egocentradas (uno que se enfoca en la red amplia del conjunto de sus relaciones y otro, 
en la red de amistades) y con el estudio sociocéntrico de las aulas de cada instituto. Otros instrumentos cuantitativos incluyeron un cuestionario general y un cuestionario de percepción de apoyo social. La aproximación cualitativa también se ubicó en este estudio en un diseño con una secuencia (QUAN $\rightarrow$ cual) e identificó las cohortes que año tras año superaban los cursos académicos con el seguimiento a detalle de sus itinerarios académicos. Se realizaron entrevistas a estudiantes que abandonaron el sistema, al profesorado referente en cada instituto, a los técnicos y profesionales de servicios municipales de apoyo educativo, y grupos de discusión con los jóvenes que mostraban persistencia.

Por la temática del presente artículo, la técnica de recogida y análisis de datos que resaltamos aquí fue un procedimiento mixto de recogida de datos cuantitativos y cualitativos implementado al final del estudio que combinaba información obtenida con el cuestionario de red (perspectiva theory driven) y minirrelatos biográficos (grounded theory). Con los 20 estudiantes que completaron los estudios postobligatorios, elaboramos una técnica que denominamos entrevista egonarrativa (Cano, Sandín, Sánchez \& Torrent, 2014). A cada estudiante, se le mostraba la red personal graficada al inicio del estudio y la de su momento actual (obtenida in situ en el momento de la entrevista con el cuestionario de redes) y se dialogaba sobre diversos aspectos comunes y diferentes de las mismas (estructura de la red, agentes significativos, densidad de relaciones, tipos de apoyo recibido, personas significativas incorporadas, etc.) con lo cual se creaba un contexto para la elicitación de breves relatos que mostraran aquellas personas, espacios y momentos vitales destacables en su historia de logro académico.

Figura 6

Entrevista egonarrativa. Cuestionario de redes + Relatos biográficos

\section{Modelo Teórico de Resiliencia Saavedra y Villalta (2008)}

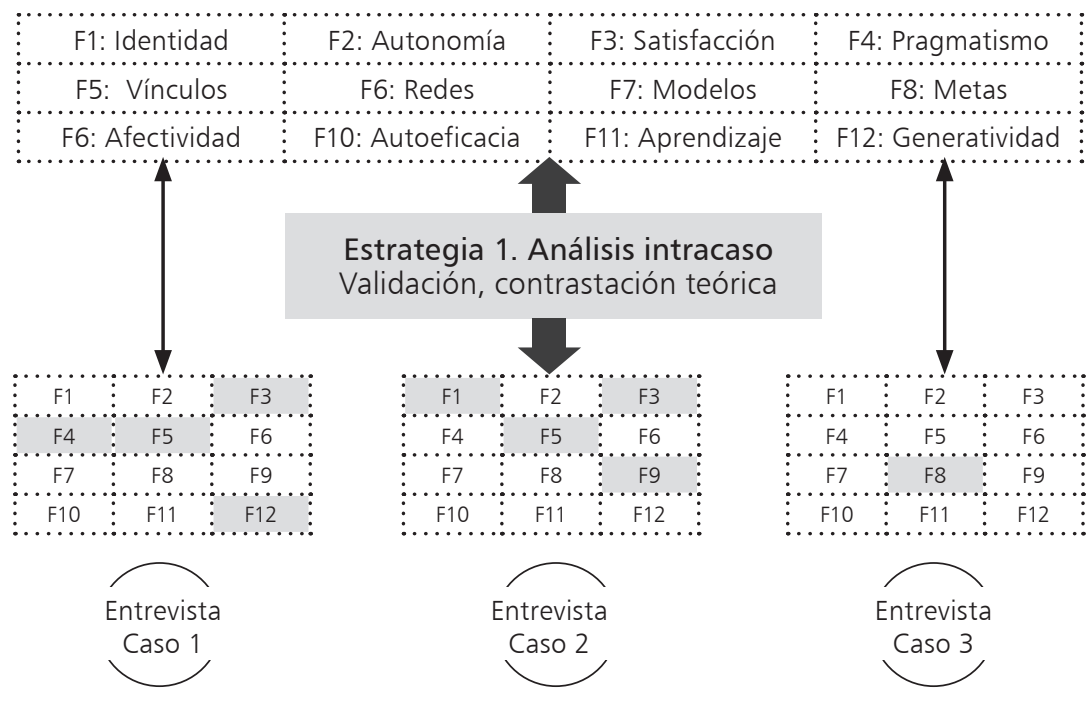

Estrategia 2. Análisis transversal Dimensiones sustantivas. Análisis temático 
El análisis de los relatos vinculados a la red personal de los estudiantes se benefició de un enfoque mixto deductivo-inductivo; por un lado, se dispone de las dimensiones de análisis establecidas de forma "deductiva" a partir del marco conceptual (figura 5) (que también sostuvieron la elaboración de los instrumentos de recogida de datos - cuestionario de redes y entrevista-) y, por otro, de las que surgen de los propios relatos, en ocasiones, al concretar o reconstruir las primeras; por ejemplo, al analizar la evolución de las redes personales de los jóvenes, constatamos que no se da la aparición de "nuevos nodos significativos", sino que hay una tendencia a la estabilidad de la red, con un cambio en la intensidad de relación o de significatividad; dicho de otro modo, se aprecian un "redescubrimiento" y una "revalorización" de nodos ya existentes en su red. El proceso de categorización se usa así también para abrir la indagación y pasar a la interpretación, y representa el vínculo decisivo entre la información y los conceptos teóricos; la descontextualización y la recontextualización ayudan a reducir y luego expandir los datos, conceptualizarlos, plantear preguntas, proporcionar respuestas provisionales sobre las relaciones entre ellos y dentro de ellos, y descubrirlas. Ciertamente, la incorporación de la perspectiva biográfico-narrativa en la exploración y análisis de los relatos ofreció una información pragmática, situacional y contextual de gran valor (Lozares \& Verd, 2008) para una mayor comprensión de los casos de éxito y de la evolución de sus sistemas relacionales.

\section{Discusión}

Considerando el desarrollo cada vez mayor de los métodos cualitativos en las ciencias sociales, hemos intentando en este artículo abordar la cuestión del uso y la generación de teoría en la investigación cualitativa. Lo discutido en este artículo es probablemente familiar a los investigadores cualitativos, particularmente el problema de la inducción-deducción o la dificultad de conducir investigaciones sin asunciones teóricas sustantivas (Bendassolli, 2013). El papel de la teoría en los estudios cualitativos es un tema complejo que ya ha sido abordado en distintas obras que muestran la continuidad de los debates y el interés actual. Vincent A. Anfara y Norma T. Mertz (2006) señalan que no hay consenso ni sobre el rol de la teoría en la investigación cualitativa, ni sobre lo que debe discutirse; según estos autores, un examen de los materiales más destacados sobre estas cuestiones deja al lector tres comprensiones diferentes: una, que la teoría tiene poca relación con la investigación cualitativa; dos, que la teoría está relacionada con la metodología elegida y las epistemologías que subyacen a la metodología y, en tercer lugar, que la teoría en la investigación cualitativa es más amplia y más dominante en su papel que la metodología. ¿Por qué es tan importante "la teoría"? Priscilla Alderson (1998) explicó que la teoría está en el corazón de la práctica, la planificación y la investigación y que todo pensamiento involucra teorías; estas influyen poderosamente en la recolección, análisis, comprensión y uso de la evidencia, es "práctico y científico" examinarlas, dice esta autora. Siguiendo a Anfara y Mertz (2006), el marco conceptual en el proceso de investigación tiene la habilidad de: 1) focalizar el estudio (orientar el análisis; establecer conceptos, constructos y proposiciones; ser consciente de la perspectiva utilizada), 2) revelar y ocultar el significado y la comprensión (las teorías pueden tanto permitirnos ver los fenómenos familiares de una manera novedosa, pero también pueden cegarnos ante aspectos del fenómeno que no forman parte de la teoría), 3) situar la investigación en una conversación académica y 4) revelar sus fortalezas y debilidades; los autores concluyen que la adopción de un marco teórico no solo guía el pensamiento del investigador acerca de un fenómeno en estudio, sino que lo obliga a asegurar que la metodología, los datos y los análisis son congruentes con la teoría. Aunque algunos enfoques cualitativos (como la teoría fundamentada y, en algunos casos, la fenomenología) requieren que los investigadores suspendan a priori los compromisos teóricos, dice Margarete Sandelowski (1993) que ello no es mandato para ignorar la literatura relevante. Debemos arriesgarnos más en el desarrollo de teorías (Morse, 2003), no abandonar prematuramente el compromiso intelectual y hacer más trabajo teórico (Cisneros-Puebla, 2004), también pensar desde múltiples perspectivas (Jackson \& Mazzei, 2012). La investigación cualitativa está llamada a superar los enfoques descriptivistas, a usar la teoría para pensar con los datos, usar los datos para pensar con teoría.

\section{Conclusiones}

Que un proyecto de investigación esté guiado por una theoretical drive se refiere a

la dirección general, inductiva o deductiva, de la investigación, es decir, si el proyecto es de descubrimiento o de prueba. La investigación cualitativa generalmente, pero no siempre, usa métodos inductivos, y la cuantitativa, o de prueba de teorías, de nuevo por lo general, pero no siempre usa métodos deductivos (Morse, Niehaus, Wolfe \& Wilkins, 2006, p. 280).

En este artículo, hemos descrito algunas de las decisiones de diseño y fundamentación teórica de dos 
investigaciones theory driven sobre el éxito escolar de jóvenes de origen migratorio basadas en el método de estudio de casos, con un diseño mixto secuencial (QUAN $\rightarrow$ qual) (Creswell \& Plano-Clark, 2007). Janice M. Morse, Linda Niehaus, Ruth R. Wolfe y Seanne Wilkins (2006) advierten que desatender el theoretical drive de un proyecto con método mixto, conduce a un diseño sin ajuste, con fases deshilvanadas o desconectadas, sin especificar el ritmo en los componentes de la investigación. Anne MacFarlane y Mary O'Reilly-de Brún (2011) señalan que los diseños de investigación cualitativa theory driven son útiles para sensibilizar a los investigadores hacia conceptos o procesos que no necesariamente habrían identificado a través de procesos inductivos, y añaden que hay pocas aportaciones que discutan sobre cómo los investigadores deciden o no aplicar estos enfoques y sus resultados. El papel del marco conceptual fue importante en nuestros estudios para modular a través de diversas teorías y conceptos los intereses indagativos sobre el fenómeno, afinar y concretar las cuestiones específicas y los objetivos de cada uno, así como para orientar el diseño y la construcción de instrumentos de recogida de datos. Para el equipo de investigación fue vital compartir y desarrollar una "conciencia teórica" común que en equilibrio con la búsqueda de nuevo conocimiento se benefició de un diálogo con los marcos teóricos existentes (Andersen \& Kragh, 2009).

\section{Agradecimientos}

A los equipos directivos y coordinadores de los Institutos participantes por abrirnos las puertas de sus centros y darnos acceso a su información. Al profesorado de secundaria por permitirnos interrumpir sus clases y colaborar en la recogida de datos. Al personal becario y colaborador de ambas investigaciones. A la Agència de Gestió d'Ajuts Universitaris i de Recerca, y al Ministerio de Economía y Competitividad, por la concesión de los proyectos respectivos y el apoyo económico facilitado. A todo el alumnado de cuarto curso de la ESO y de Bachillerato por su buena disposición a participar en los estudios.

\section{Sobre la autora}

María Paz Sandín-Esteban es doctora en pedagogía por la Universitat de Barcelona. Profesora titular del Departamento de Métodos de Investigación y Diagnóstico en Educación en la Facultat d'Educació de la Universitat de Barcelona. Coordinadora del GREDI (Grupo de Investigación en Educación Intercultural) http://www.ub.edu/gredi/

\section{Referencias}

Alderson, P. (1998). The Importance of Theories in Health Care. British Medical Journal, BMJ, 317 (7164), 1007-1010. DOI: https://doi.org/10.1136/ bmj.317.7164.1007. Disponible en: https://www. jstor.org/stable/25180641?seq=1\#page_scan_ tab_contents

Andersen, P. H. \& Kragh, H. (2010). Sense and Sensibility: Two Approaches for Using Existing Theory in Theory-Building Qualitative Research. Industrial Marketing Management, 39 (1), 49-55. https:// doi.org/10.1016/j.indmarman.2009.02.008

Andréu-Abela, J.; García-Nieto, A. \& Pérez-Corbacho, A. M. (2007). Evolución de la teoría fundamentada como técnica de análisis cualitativo. Madrid: Centro de Investigaciones Sociológicas, CIS.

Anfara, V. A. \& Mertz, N. T. (eds.) (2006). Theoretical Frameworks in Qualitative Research. Thousand Oaks, California: Sage.

Bartolomé-Pina, M. (2017). Diversidad educativa. ¿Un potencial desconocido? Revista de Investigación Educativa, 35 (1), 15-33. DOI: http:// dx.doi.org/10.6018/rie.35.1.275031. Disponible en: http://revistas.um.es/rie/article/viewFile/27 $5031 / 203511$

Bendassolli, P. F. (2013). Theory Building in Qualitative Research: Reconsidering the Problem of Induction. Forum Qualitative Sozialforschung / Forum: Qualitative Social Research, 14 (1). doi: http:// dx.doi.org/10.17169/fqs-14.1.1851. http://www. qualitative-research.net/index.php/fqs/article/ view/1851/3499

Boada, C.; Herrera, D.; Mas, E.; Miñarro, E.; Olivella, M.; Riudor, X.; Calero, J.; Choi, A. \& Escardíbul, J. O. (2011). Informe sobre el risc de fracàs escolar a Catalunya. Barcelona: Consell de Treball Económic i Social de Catalunya (CTESC), Collecció Estudis i Informes, 26. Disponible en: http://ctesc. gencat.cat/doc/doc_53893194_1.pdf

Bradbury-Jones, C.; Taylor, J. \& Herber, O. (2014). How Theory is Used and Articulated in Qualitative Research: Development of a New Typology. Social Science \& Medicine, 120, 135-141. https://doi. org/10.1016/j.socscimed.2014.09.014

Burns, N. (1989). Standards for Qualitative Research. Nursing Science Quarterly, 2 (1), 44-52. https:// doi.org/10.1177/089431848900200112

Burt, R. S. (1992). Structural Holes. The Social Structure of Competition. Cambridge, Massachusetts: Harvard University Press.

Cano, A. B.; Sandín, M. P.; Sánchez, A. \& Torrent, I. (2014). Using Narratives to Study the Impact of Social Networks on the Educational Paths of Immigrant Students. Barcelona: Dipòsit Digital de la 
Universitat de Barcelona. Disponible en: http:// hdl.handle.net/2445/55852.

Cisneros-Puebla, C. A. (2004). "Let's Do More Theoretical Work...". Janice Morse in Conversation with César A. Cisneros-Puebla. Forum Qualitative Sozialforschung / Forum: Qualitative Social Research, 5 (3), article 33. http://nbn-resolving. de/urn:nbn:de:0114-fqs0403331, http://www. qualitative-research.net/index.php/fqs/article/ view/556/1203

Coffey, A. \& Atkinson, P. (1996). Making Sense of Qualitative Data. Complementary Research Design. Thousand Oaks, California: Sage.

Creswell, J. W. (2007). Qualitative Inquiry \& Research Design: Choosing among Five Approaches. $2^{\text {nd }}$ ed., Thousand Oaks, California: Sage Publications. Disponible en: https://charlesbicken heuserdotcom.files.wordpress.com/2015/03/ creswell_2007_qualitative_inquiry_and_research_design_choosing_among_five_ approaches_2nd_edition.pdf

Creswell, J. W. \& Plano-Clark, V. (2007). Designing and Conducting Mixed Methods Research. Thousand Oaks, California: Sage.

Cuesta-Benjumea, C. de la (2008). ¿Por dónde empezar?: la pregunta en investigación cualitativa. Enfermería Clínica, 18 (4), 205-210. Disponible en: http://rua.ua.es/dspace/handle/10045/17048

Denzin, N. K. \& Lincoln, Y. S. (eds.) (1994). Handbook of Qualitative Research. London: Sage.

Eisenhardt, K. M. \& Graebner, M. E. (2007). Theory Building from Cases: Opportunities and Challenges. Academy of Management Journal, 50 (1), 25-32. Disponible en: https://aom.org/ uploadedFiles/Publications/AMJ/Eisenhart.Grae bner.2007.pdf

Fergus, S. \& Zimmerman, M. A. (2005). Adolescent Resilience: A Framework for Understanding Healthy Development in the Face of Risk. Annual Review of Public Health, 26, 399-419. Disponible en: https:// www.annualreviews.org/doi/pdf/10.1146/annu rev.publhealth.26.021304.144357

García-Ramírez, M.; Martínez-García, M. F. \& AlbarMarín, M. J. (2002). La elección de fuentes de apoyo social entre inmigrantes. Psicothema, 14 (2), 369-374. Disponible en: https://idus.us.es/ xmlui/handle/11441/56453

Gibbs, G. (2012). El análisis de datos cualitativos en investigación cualitativa. Madrid: Morata.

Glaser, B. G. \& Strauss, A. L. (1967). The Discovery of Grounded Theory: Strategies for Qualitative Research. Chicago: Aldine.

Granovetter, M. (1973). The Strength of Weak Ties. American Journal of Sociology, 78, 1360-1380.
Disponible en: https://www.cs.cmu.edu/ jure/ pub/papers/granovetter73ties.pdf

Grotberg, E. (1995). A Guide to Promoting Resilience in Children: Strengthening the Human Spirit. The Hague: Bernard van Leer Foundation. Disponible en: https://bibalex.org/baifa/Attachment/Docu ments/115519.pdf

Guzmán-Valenzuela, C. M. (2014). Polos epistemológicos, uso y construcción de teoría en investigación cualitativa en educación. Magis, Revista Internacional de Investigación en Educación, 7 (14), 15-28. DOI: http://dx.doi.org/10.11144/Ja veriana.M7-14.PEUC. Disponible en: http://revistas. javeriana.edu.co/index.php/MAGIS/article/ view/11852/pdf

Jackson, A. Y. \& Mazzei, L. A. (2012). Thinking with Theory in Qualitative Research. Viewing Data across Multiple Perspectives. New York: Routledge.

Kaplan, H. B. (1999). Towards an Understanding of Resilience: A Critical Review of Definitions and Models, en M. D. Glantz \& J. L. Johnson (eds.). Resilience and Development. Positive Life Adaptations, 17-83. Nueva York: Kluwer Academic/ Plenum Press.

Lakoff, G. \& Johnson, M. (2004). Metáforas de la vida cotidiana. $6^{a}$ ed., Madrid: Cátedra.

Lin, N. (1999). Building a Network Theory of Social Capital. Connections, 22 (1), 28-51. Disponible en: http://www.insna.org/PDF/Keynote/1999.pdf

Llewellyn, S. (2003). What Counts as "Theory" in Qualitative Management and Accounting Research? Introducing Five Levels of Theorizing. Accounting, Auditing \& Accountability Journal, 16 (4), 662-708. DOI: 10.1108/09513570310492344

Lozares, C. \& Verd, J. M. (2008). La entrevista biográfico-narrativa como expresión contextualizada, situacional y dinámica de la red socio-personal. REDES, Revista Hispana para el Análisis de Redes Sociales, 15 (6), 95-125. Disponible en: www.raco.cat/index.php/Redes/article/down load/121674/168137

Luthar, S. S. (2003) Resilience and Vulnerability. Adaptation in the Context of Childhood Adversities. Cambridge: Cambridge University Press.

MacFarlane, A. \& O’Reilly-de Brún, M. (2011). Using a Theory-Driven Conceptual Framework in Qualitative Health Research. Qualitative Health Research, 22 (5), 607-618. https://doi.org/10. $1177 / 1049732311431898$

Masten, A. S. (2001). Ordinary Magic: Resilience Processes in Development. American Psychologist, 56 (3), 227-238. http://dx.doi.org/10.1037/0003$066 \times .56 .3 .227$ 
Medina, J. L. (2014). El proceso de comprensión en el análisis de datos cualitativos en educación. Magis. Revista Internacional de Investigación en Educación, 7 (14), 39-54. DOI: http://dx.doi.org/10.11144/ Javeriana.M7-14.EPCA. Disponible en: http://revistas.javeriana.edu. co/index.php/MAGIS/article/view/11855/pdf_1

Meyer, S. \& Ward, P. (2014). 'How to' Use Social Theory within and Throughout Qualitative Research in Healthcare Contexts. Sociology Compass, 8 (5), 525-539. https://doi.org/10.1111/soc4.12155

Molina, J. L. (2001). El análisis de redes sociales. Una introducción. Barcelona: Edicions Bellaterra.

Morse, J. M. (1997). Considering Theory Derived from Qualitative Research. En J. M. Morse (ed.). Completing a Qualitative Project. Details and Dialogue, 163-189. Thousand Oaks, California: Sage.

Morse, J. M. (2003). "Emerger de los datos": los procesos cognitivos del análisis en la investigación cualitativa. En J. M. Morse (ed.). Asuntos críticos en los métodos de investigación cualitativa, 29-55. Medellín: Editorial Universidad de Antioquia.

Morse, J. M.; Niehaus, L.; Wolfe, R. R. \& Wilkins, S. (2006). The Role of the Theoretical Drive in Maintaining Validity in Mixed-Method Research. Qualitative Research in Psychology, 3 (4), 279-291.

Organisation for Economic Cooperation and Development, OECD (2011). Education at a Glance 2011: OECD Indicators. Paris: OECD Publishing. http://dx.doi.org/10.1787/eag-2011-en. Disponible en: https:// www.oecd-ilibrary.org/education/education-at-a-glance-2011_eag2011-en

Punch, K. F. (2005). Introduction to Social Research. London: Sage.

Resilience Research Center (s.f.). Interview Guide. Disponible en: http:// www.resilienceresearch.org/research/resources/tools/29-inter view-guide

Richardson, A. J. (2017). The Discovery of Cumulative Knowledge: Strategies for Designing and Communicating Qualitative Research. Accounting, Auditing \& Accountability Journal, 31 (2), 563-585. Disponible en: https://scholar.uwindsor.ca/odettepub/126

Saavedra-Guajardo, E. \& Villalta-Paucar, M. (2008). Escala de resiliencia para jóvenes y adultos (SV-RES). Santiago de Chile: Centro de Estudios y Atención del Niño y la Mujer, CEANIM. Disponible en: http:// wWw.academia.edu/26343874/Saavedra_-Villalta_SV-RES60_ES CALA_DE_RESILIENCIA_SV-RES

Sandelowski, M. (1993). Theory Unmasked: the Uses and Guises of Theory in Qualitative Research. Research in Nursing and Health, 16 (3), 213218. https://doi.org/10.1002/nur.4770160308

Sandín-Esteban, M. P. \& Sánchez-Martí, A. (2013). Resiliencia y persistencia académica en estudiantes inmigrantes. Revista Iberoamericana de Educación (OEI), 62 (1), 1-11. Disponible en: https://rieoei.org/ RIE/article/view/890

Scott, J. \& Carrington, P. J. (2011). The SAGE Handbook of Social Network Analysis. Los Angeles: Sage.

Serra, C. \& Palaudàrias, J. M. (2010). Continuar o abandonar. L'alumnat estranger a l'educació secundària. Barcelona: Fundació Jaume Bofill. Disponible en: http://www.fbofill.cat/sites/default/files/519.pdf

Stake, R. E. (1995). The Art of Case Study Research. Thousand Oaks, California: Sage.

Stanton-Salazar, R. D. (1997). A Social Capital Framework for Understanding the Socialization of Racial Minority Children and Youths. 
Harvard Educational Review, 67 (1), 1-41. https://doi.org/10.17763/ haer.67.1.140676g74018u73k

Steenhuis, H. J. \& de Bruijn, E. J. (2006). Building Theories from Case Study Research: the Progressive Case Study. In OM in the New World Uncertainties. Proceedings (CD-ROM) of the $17^{\text {th }}$ Annual Conference of POMS, 28 April - 1 May 2006, Boston, USA. Production and Operations Management Society (POMS). Disponible en: https://ris. utwente.nl/ws/portalfiles/portal/5607911

Strauss, A. \& Corbin, J. (1990). Basics of Qualitative Research. Grounded Theory Procedures and Techniques. Thousand Oaks, California: Sage.

Trinidad-Requena, A.; Carrero-Planes, V. \& Soriano-Miras, R. M. (2006). Teoría Fundamentada "Grounded Theory". La construcción de la teoría a través del análisis interpretacional. Madrid: Centro de Investigaciones Sociológicas, CIS, Cuadernos Metodológicos 37.

Yin, R. K. (1994). Case Study Research. Design and Methods. 2 ed., Thousand Oaks, California: Sage. 